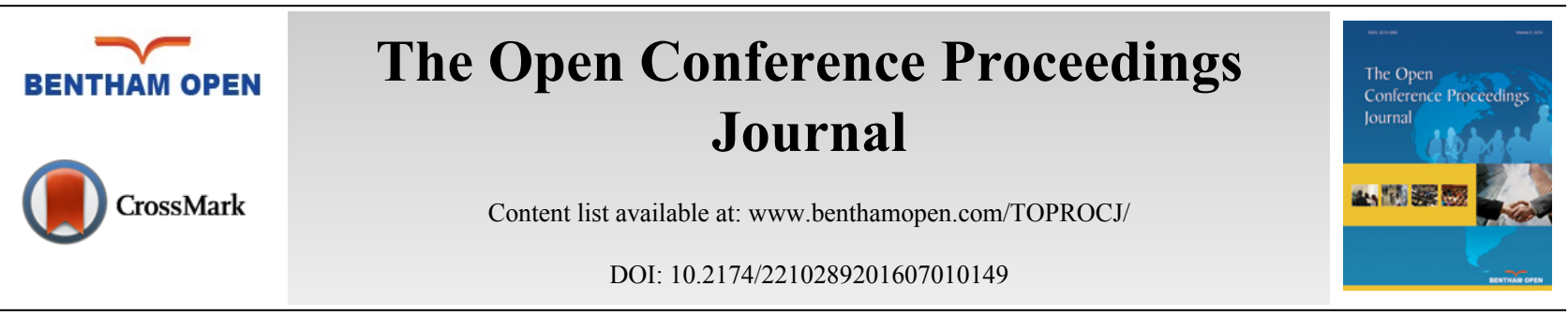

CASE REPORT

\title{
EMDR for Treatment of Anxiety Disorder in a Patient with Intellectual Disability
}

\author{
Farshad Shaddel ${ }^{*}$ \\ Learning Disability, NHS, UK; University of Oxford, UK; Higher Education Academy, UK
}

Received: February 18, 2016

Revised: October 02, 2016

Accepted: October 20, 2016

\begin{abstract}
Eye Movement Desensitization and Reprocessing (EMDR) is a structured, eight-phase, mainly non-verbal psychotherapeutic approach that is developed to resolve symptoms that have stemmed from disturbing and unprocessed life experiences. EMDR is well known for the treatment of Post-Traumatic Stress Disorder (PTSD). However, modified protocols have been developed for the treatment of other anxiety and mood disorders; especially if rooted from a traumatic experience in the past.

People with Intellectual Disability (ID) are more likely to suffer from adverse experiences during their lives and psychiatric consequences are common among them. In addition, they are more likely to have difficulty in verbal communication which is a requirement in other psychological interventions but less so in EMDR. All these point towards EMDR as a potential add-on tool in this group of clients.

Unfortunately there is limited, yet encouraging, published literature about the use of EMDR in people with ID. In this paper, I will report a successful use of EMDR for the treatment of PTSD and Obsessive Compulsive Disorder (OCD) in a 35 year old patient with moderate ID and then use this to discuss the advantages and disadvantages of using EMDR for people with ID in a wider context.
\end{abstract}

Keywords: Anxiety disorder, EMDR, Intellectual disability, Learning disability, Mental retardation, Obsessive compulsive disorder, OCD, PTSD.

\section{BACKGROUND}

Eye Movement Desensitization and Reprocessing (EMDR) is a structured, mainly non-verbal, eight-phase psychotherapeutic approach that has been developed to resolve symptoms resulting from disturbing and unprocessed life experiences [1,2]. EMDR is well known for the treatment of Post-Traumatic Stress Disorder (PTSD) [3 - 8]. However, modified protocols have been developed for the treatment of other anxiety and mood disorders specially if stemmed from a traumatic experience in the past $[9,10]$. The main difference between modified protocol and standard eight-phase protocol is the three-layered approach to past, present and future. This is usually attributed to the model introduced by Dr Parnell [9] to address the role of current triggers and future coping mechanisms in the successful treatment of anxiety disorders as well as the original traumatic event; usually called "Touchstone memory". It is not uncommon to observe that patients have forgotten the original memory due to the significance of current triggers. In such circumstances, different techniques such as "Affect scan" and "Float back" are used to identify the earliest experience of such negative thoughts or feelings in order to discover the "Touchstone memory" [9].

People with intellectual disability (ID) are more likely to suffer from adverse experiences in their lives and psychiatric consequences are common among them [11 - 13]. In addition, they are more likely to have difficulty verbally communicating which is more important in other psychological interventions but less so in EMDR [2]. All these facts point towards EMDR as a potential adds-on tool should it's effectiveness in this group of clients be proven.

\footnotetext{
* Address correspondence to this author at the Berkshire Healthcare NHS Foundation Trust, Prospect Park Hospital, Reading, RG30 4EJ, UK; Tel: 0044(118)9605114; Fax: 0044(118)9605115; E-mails: f_shaddel@yahoo.com; farshad.shaddel@berkshire.nhs.uk
} 
Unfortunately, there are only a few, but still encouraging case reports about the use of EMDR in people with ID $[2,14,15]$.

Of course, the efficacy of EMDR in this group of clients can only be established for sure through well designed, randomised clinical trials. However, reporting and publishing the successful application of a therapeutic tool in new group of patients is still very important. This will encourage researchers and organisations to design and invest in conducting such clinical trials which are usually time-consuming and costly.

In this manuscript, I am reporting the successful use of EMDR for the treatment of PTSD and then Obsessive Compulsive Disorder (OCD) in a patient with moderate ID. Patient has given consent for reporting his case.

\section{CASE PRESENTATION}

Mr. X is a 35 year old gentleman with a moderate ID and a long history of depression, anxiety and severe OCD. When I took over the job of his care in August 2013, he would spend most of the day lying on a sofa under a duvet. He was unable to move around the house due to lengthy rituals when passing a door and each shower took five hours. The initial assessment revealed the presence of flash-backs and nightmares of a bullying event that occurred when Mr. X was 15. A diagnosis of PTSD was established at this stage as well. He misused alcohol to be able to socialise and go to sleep. In return alcohol would make him more depressed, suicidal and occasionally aggressive toward others. He would respond partially to Fluoxetine $60 \mathrm{mg}$ but he was unable to tolerate that dose due to its side effects.

After I built up a therapeutic rapport and motivated him to cut his alcohol use, he was considered a suitable candidate for EMDR. He received the first five sessions of EMDR from April to May 2014. During the treatment, his memory of being bullied at age 15 was processed by a standard 8-phase protocol and rapid eye movements.

Flashbacks and nightmares disappeared during the treatment and the quality of sleep, mood and general well-being improved. He stopped misusing alcohol and binge drinking as well but there was no significant change in the severity of OCD. His pre-treatment Hamilton Anxiety and Depression Scale (HADS) were 14 (Depression) and 16 (Anxiety) and they dropped to 4 and 12 respectively after treatment Fig. (1). Given the encouraging response of PTSD to EMDR, it was agreed to give another set of EMDR for his OCD and anxiety in due course.

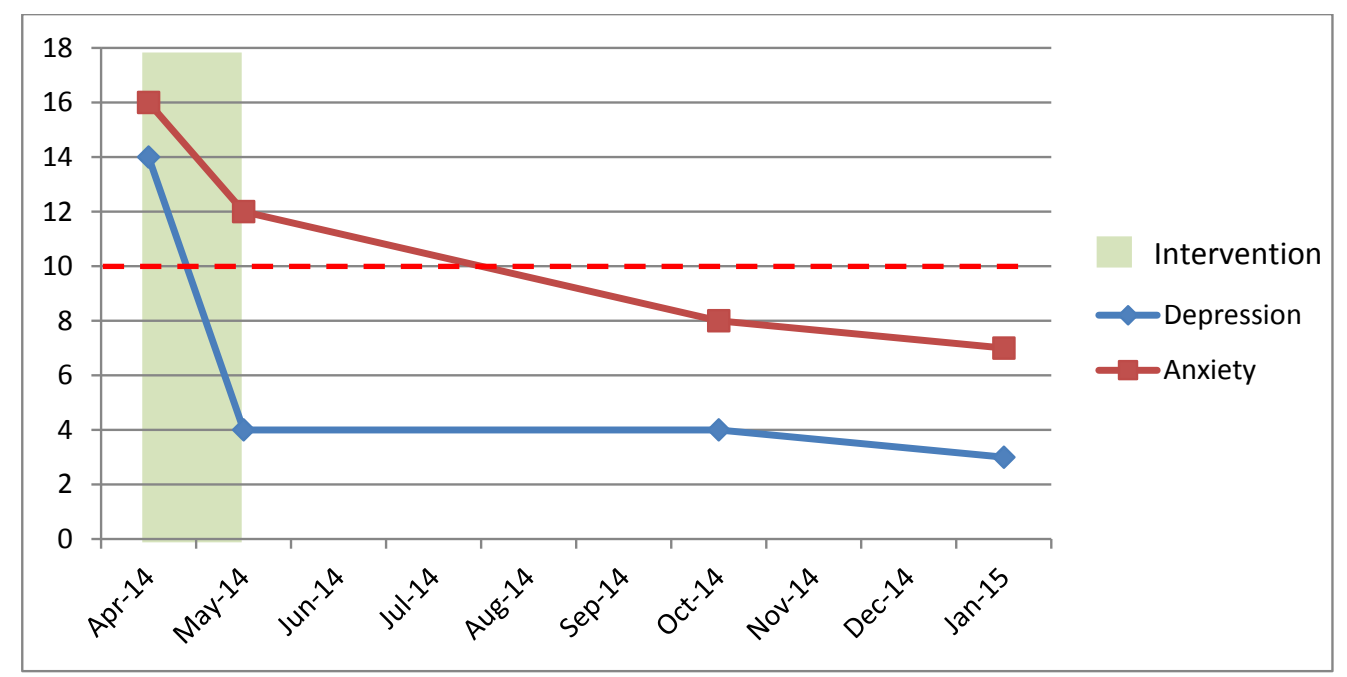

Fig. (1). HADS score pre and post treatment; 0-7 (Normal), 8-10 (Borderline) and 11-21 (Abnormal).

He continued to do well and improvement in his HADS score sustained; it was 4 (Depression) and 8 for anxiety in October 2014 although OCD was still present. He scored 55 out of maximum of 72 with a cut-off of 21 on the Obsessive Compulsive Inventory - Revised (OCI-R) scale which suggested severe OCD. His main problems were that of taking long showers, counting before doing certain functions, obsessive slowness and fear of contamination. He could not remember how the illness started.

Mr. X received another 6 weekly sessions of EMDR between Oct 2014 and January 2015. During this treatment; the touchstone memory was identified by the "float-back" technique. He remembered for the first time that his illness started after a high fever and being sick when he was 10 years old. He felt for the first time in his delirious state that his 
face was dirty because of vomiting and he had to wash it constantly. His OCD remained with him after that, however; the presentations and rituals had changed slightly during years.

An anxiety protocol was used and his touchstone memory, the worst memory, the current triggers and the future template were processed by rapid eye movements. Mr. X reported progressive improvement in his anxiety and rituals. He stopped counting from the third session and he was able to clean the house and cook in his kitchen at the end. The shower time reduced to two hours. HADS and OCI-R were repeated in January 2015. He scored 3 (Depression) and 7 (Anxiety) on HADS and 32 on OCI-R (Figs. 1 and 2).

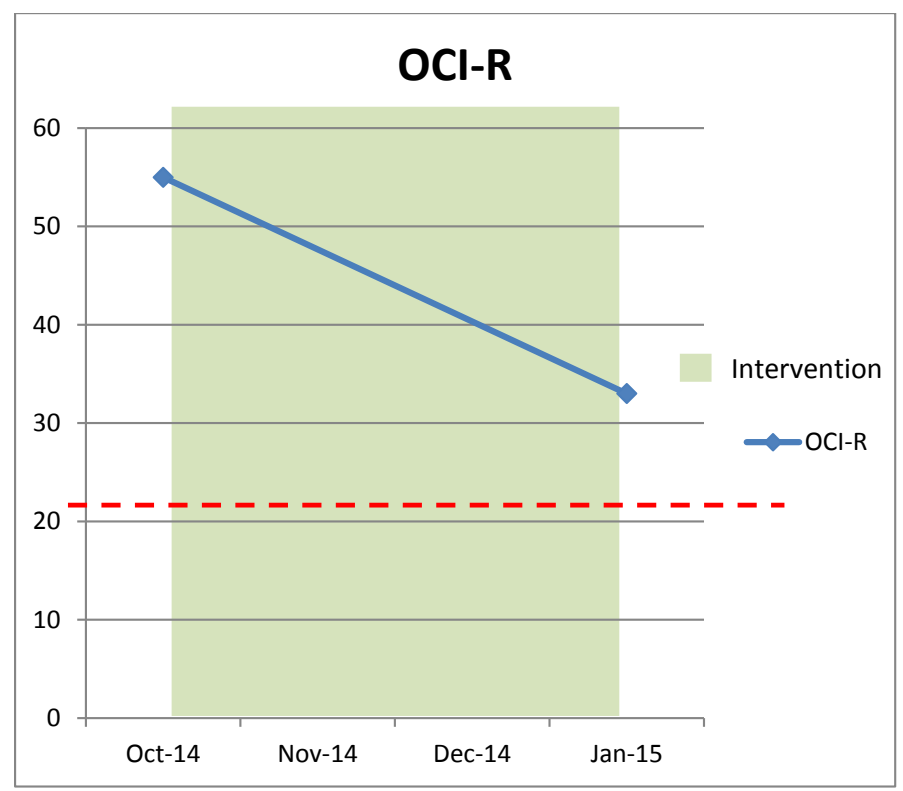

Fig. (2). OCI-R pre and post treatment; Maximum 72 and cut off of 21.

\section{DISCUSSION AND RECOMMENDATIONS}

EMDR seems to be a useful and effective add-on method of treatment for psychiatric disorders in people with ID; especially if the problem began due to a traumatic experience. Relatively short duration of intervention, minimal reliance on the verbal abilities and an absence of interaction with other psychiatric interventions could be considered as it's advantages in this group of client. However, its efficacy still needs to be proven in controlled studies but nevertheless it could be a viable option in a case that has exhausted other pharmacological and psychological interventions.

This case study suggests that proper assessment of suitability and building up therapeutic relations with patient and creativity in using the standard protocols to adapt to the specific needs of patients with ID would improve the chance of a successful treatment which is in line with other psychological interventions [16].

\section{CONFLICT OF INTEREST}

The author confirms that this article content has no conflict of interest.

\section{ACKNOWLEDGEMENTS}

Declared none.

\section{REFERENCES}

[1] Shapiro, F. The role of eye movement desensitization and reprocessing (EMDR) therapy in medicine: addressing the psychological and physical symptoms stemming from adverse life experiences. Perm. J., 2014, 18(1), 71-77. [http://dx.doi.org/10.7812/TPP/13-098] [PMID: 24626074]

[2] Mevissen, L.; de Jongh, A. PTSD and its treatment in people with intellectual disabilities: a review of the literature. Clin. Psychol. Rev., 2010, $30(3), 308-316$.

[http://dx.doi.org/10.1016/j.cpr.2009.12.005] [PMID: 20056303] 
[3] Lee, C.; Gavriel, H.; Drummond, P.; Richards, J.; Greenwald, R. Treatment of post-traumatic stress disorder: A comparison of stress inoculation training with prolonged exposure and eye movement desensitisation and reprocessing. J. Clin. Psychol., 2002, 58, $1071-1089$. [http://dx.doi.org/10.1002/jclp.10039] [PMID: 12209866]

[4] Marcus, S.; Marquis, P.; Sakai, C. Controlled study of treatment of PTSD using EMDR in an HMO setting. Psychotherapy, 1997, 34, 307-315.

[http://dx.doi.org/10.1037/h0087791]

[5] Rothbaum, B.O. A controlled study of eye movement desensitization and reprocessing in the treatment of posttraumatic stress disordered sexual assault victims. Bull. Menninger Clin., 1997, 61(3), 317-334.

[PMID: 9260344]

[6] Ironson, G.; Freund, B.; Strauss, J.L.; Williams, J. Comparison of two treatments for traumatic stress: a community-based study of EMDR and prolonged exposure. J. Clin. Psychol., 2002, 58(1), 113-128. [http://dx.doi.org/10.1002/jclp.1132] [PMID: 11748600]

[7] National Collaborating Centre for Mental Health. Post traumatic stress disorder (PTSD). The management of adults and children in primary and secondary care, London: National Institute for Clinical Excellence. Available from: https://www.nice.org.uk/guidance/cg26/ evidence/full-guideline-including-appendices-113-193442221 [Accessed October 2, 2016].

[8] American Psychiatric Association. Practice Guideline for the Treatment of Patients with Acute Stress Disorder and Posttraumatic Stress Disorder, Arlington, VA: American Psychiatric Association Practice Guidelines. Available from: http://psychiatryonline.org/pb/assets/ raw/sitewide/practice_guidelines/guidelines/acutestressdisorderptsd.pdf [Accessed October 2, 2016].

[9] Parnell, L. A Therapist's Guide to EMDR: Tools and Techniques for Successful Treatment, $1^{\text {st }}$ ed.; WN Norton \& Company: New York, London, 2007.

[10] Bae, H.; Kim, D.; Ahn, J. A case series of posttraumatic obsessive-compulsive disorder: A six month follow-up evaluation. J. Korean Neuropsychiatr. Assoc., 2006, 45(5), 476-480.

[11] Bramston, P.; Fogarty, G.; Cummins, R.A. The nature of stressors reported by people with an intellectual disability. J. Appl. Res. Intellect. Disabil., 1999, 18(6), 435-456.

[12] Fogarty, G.J.; Bramston, P.; Cummins, R.A. Validation of the Lifestress Inventory for people with a mild intellectual disability. Res. Dev. Disabil., 1997, 18(6), 435-456.

[http://dx.doi.org/10.1016/S0891-4222(97)00021-8] [PMID: 9403927]

[13] Hartley, S.L.; MacLean, W.E., Jr Perceptions of stress and coping strategies among adults with mild mental retardation: insight into psychological distress. Am. J. Ment. Retard., 2005, 110(4), 285-297. [http://dx.doi.org/10.1352/0895-8017(2005)110[285:POSACS]2.0.CO;2] [PMID: 15941365]

[14] Giltaij, H. As if a vacuum cleaner went through my head. EMDR in people with visual and intellectual disabilities. In: Tijdschrift voor Kinder-\& Jeugdpsychotherapie; , 2004; 3, pp. 1-97.

[15] Mevissen, L.; Lievegoed, R.; Seubert, A.; De Jongh, A. Do persons with intellectual disability and limited verbal capacities respond to trauma treatment? J. Intellect. Dev. Disabil., 2011,36(4), 274-279. [http://dx.doi.org/10.3109/13668250.2011.621415] [PMID: 22017196]

[16] Ardito, R.B.; Rabellino, D. Therapeutic alliance and outcome of psychotherapy: historical excursus, measurements, and prospects for research. Front. Psychol., 2011, 2, 270.

[http://dx.doi.org/10.3389/fpsyg.2011.00270] [PMID: 22028698]

(C) Farshad Shaddel; Licensee Bentham Open

This is an open access article licensed under the terms of the Creative Commons Attribution-Non-Commercial 4.0 International Public License (CC BY-NC 4.0) (https://creativecommons.org/licenses/by-nc/4.0/legalcode), which permits unrestricted, non-commercial use, distribution and reproduction in any medium, provided the work is properly cited. 